\title{
Age differences on the California Card Sorting Test: Implications for the assessment of problem solving by the elderly
}

\author{
WILLIAM W. BEATTY \\ University of Oklahoma Health Sciences Center, Oklahoma City, Oklahoma
}

\begin{abstract}
The performance of young, middle-aged, and old groups of healthy normal controls was compared on two tests of problem solving: the Wisconsin and the California Card Sorting Tests (WCST and CCST). In agreement with previous studies of the WCST, subjects 60 years of age and older achieved fewer categories and made more perseverative responses and errors than did younger subjects. However, the number of categories achieved and the measures of perseveration were so highly and negatively correlated that they are not likely to be independent. On the CCST, which provides multiple measures of performance, the old subjects generated and identified fewer correct concepts and executed concepts less accurately when guided by abstract cues, but they showed increased perseveration on only one of three measures. Furthermore, the old subjects executed concepts in response to explicit cues and explained the correct sorts that they produced as accurately as did the younger subjects. Recent studies demonstrate that distinctive patterns of problem-solving impairment on the CCST are exhibited by patients with Parkinson's disease, multiple sclerosis, chronic alcoholism, or schizophrenia, although performance by patients with these disorders is qualitatively indistinguishable on the WCST. Since the CCST is about twice as sensitive as the WCST for detecting impairment in patients with Parkinson's disease, the CCST may prove especially useful for evaluating problem solving in the elderly.
\end{abstract}

The assessment of problem solving is an important component of any thorough neuropsychological evaluation. The widely used Category Test (Reitan \& Wolfson, 1985) has the disadvantage of yielding only a single summary score and therefore provides little information about why patients perform poorly. The Wisconsin Card Sorting Test (WCST) (Heaton, 1981) provides several different measures of performance that ostensibly offer insight into the nature of impaired problem solving. However, the consistently high negative correlations between the measure of concept attainment (categories achieved) and measures of perseveration (Beatty \& Monson, 1990, in press) make it clear that these indexes are not independent. Furthermore, a subject who performs poorly, but matches cards randomly, will exceed the recommended cutoff score for impairment on the perseverative response measure (Beatty \& Monson, 1990).

Recently, Delis, Bihrle, Janowsky, Squire, and Shimamura (1989) have devised the California Card Sorting Test (CCST), which provides separate measures of concept generation, concept identification, and concept execution, as well as several different measures of verbal and nonverbal perseverative responding. In addition, after subjects have sorted cards according to a rule, they are required to provide a verbal explanation of the rule (or

Correspondence and reprint requests should be addressed to the author at the Department of Psychiatry and Behavioral Sciences, University of Oklahoma Health Sciences Center, Suite 410, Rogers Building, P.O. Box 26901, Oklahoma City, OK 73190. principle) that they have used to generate the sort. This feature of the CCST yields a measure of the quality of verbal explanations for correct sorting behavior which is relatively independent of the number of correct sorting responses (concepts) actually generated. Thus, a subject may produce a correct concept (as inferred from the nonverbal sorting response) and be quite unable to articulate the "principle" that guided his/her behavior.

Delis, Squire, Bihrle, and Massman (1992) have reported that patients with acquired lesions of the frontal lobes or with alcoholic Korsakoff's syndrome generated, identified, and executed fewer correct concepts but made more perseverative responses than did normal controls. The frontal-lobe and Korsakoff patients also provided inadequate verbal explanations for the correct sorts that they did achieve. By contrast, a mixed group of amnesic patients of other etiologies who did not show signs of frontallobe injury (by neuropsychological or neuroimaging criteria) performed almost normally.

In our own research, we have studied the performance of patients with Parkinson's disease (PD), multiple sclerosis (MS), chronic alcoholism, and schizophrenia on both the WCST and the CCST. Compared to age- and educationequated groups of normal controls, all of these patient groups performed similarly on the WCST, achieving fewer correct concepts (categories) but emitting more perseverative responses and errors.

On the CCST, however, the various patient groups exhibited distinctive patterns of impairment. Mildly demented PD patients (Beatty \& Monson, 1990) and schizophrenics 
(Beatty, Jocic, Monson, \& Katzung, in press) exhibited poorer than normal performance on measures of concept generation, identification, and execution, accompanied by increases in verbal and nonverbal perseveration and lower quality explanations for the correct sorts that they did achieve. PD patients of normal mental status exhibited no impairments on any of these measures. Patients with MS (also of normal mental status) generated and identified fewer correct concepts than did controls, but they executed concepts normally, did not perseverate, and provided normally complete verbal explanations for their correct sorting behavior (Beatty \& Monson, in press). Chronic alcoholics also generated and identified fewer correct concepts than did controls, and executed concepts normally. Unlike the MS patients, the alcoholics exhibited more verbal and nonverbal perseverations, and their verbal explanations for correct sorting responses were markedly inferior to those of controls (Beatty, Katzung, Nixon, \& Moreland, in press). Indeed, the quality of the verbal explanations offered by the alcoholics was lower than that of the mildly demented PD patients or the chronic schizophrenics, whose overall performance on other cognitive tests was substantially inferior to that of the alcoholics.

It is now widely appreciated that the average performance of normal individuals aged 60 years and older on tests such as the Category Test and the WCST is substantially inferior to that of younger persons (Heaton, Grant, \& Matthews, 1986). To allow for variation in normal performance with age, as well as education and sex, Heaton, Grant, and Matthews (1991) have recently published "norms" for the Category Test, the WCST, and a number of other tests that are widely used in clinical neuropsychology. Although the publication of these norms represents a considerable advance over the previous state of affairs when clinicians had to make their own ad hoc corrections for age and education, only one measure (perseverative responses) from the WCST is considered. Futhermore, the norms published by Heaton et al. (1991) make no allowance for the fact that performance on tests such as the WCST by groups of normal elderly subjects is much more variable than that of comparable groups of younger individuals. For example, Davis et al. (1990) found that the magnitude of withingroup variance for perserverative errors on the WCST was more than 10 times as large for the normal elderly group than for a group of younger subjects.

Because the CCST appears to possess many advantages over the Category Test and the WCST for the analysis of problem solving by various clinical groups, a direct examination of the influence of age, gender, and education on this new test seemed worthwhile. This paper reports such an analysis and provides a direct comparison of the CCST and the WCST.

\section{METHOD}

\section{Subjects}

The subjects were 31 men and 34 women who were recruited as normal controls for neuropsychological investigations of various patient populations conducted by the author. They ranged in age from 18 to 75 years and in education from 8 to 20 years (postdoctoral training was not considered in calculating education). All subjects were carefully screened to exclude individuals with histories of medical illness (e.g., diabetes), central nervous system disease or injury, major psychiatric illness, serious head injury, or drug or alcohol abuse that could affect test performance. All the retained subjects scored within normal limits on a modified version of the Mini-Mental State Exam (Folstein, Folstein, \& McHugh, 1975). The subjects were paid for their participation and provided written informed consent. The study protocol was approved by the local Institutional Review Board. To facilitate presentation of the results, the subjects were arbitrarily divided into three age groups of 21-22 individuals: young (18-34 years), middle aged (35-59 years), and old (60 years and older).

\section{Materials and Procedure}

Wisconsin Card Sorting Test. The WCST was administered and scored as described by Heaton (1981).

California Card Sorting Test. This test consists of three sets of stimulus cards. Each set contains six cards with a single word printed on each card. The cards in each set can be sorted into two groups of three cards each, on the basis of 8 different sorting rules.

For each set, the subjects were tested under three conditions, which were administered in a fixed order. The free sorting condition came first. The six cards from the first set were arranged randomly on the table in front of the subject, who was instructed to sort the cards in as many different ways as possible and that after completing a sort he/she was to explain how the two piles were different. Three minutes were allowed for this phase, and the subjects were told to work quickly.

The second condition consisted of structured sorting. In this condition, the examiner sorted the cards in each of the eight correct ways, and the subject was allowed $60 \mathrm{sec}$ to describe the rule following each sort. The third condition was cued sorting. The subjects were again asked to sort the cards into two piles of three cards each, but on this part of the test the examiner provided the rule by which the cards were to be sorted. First, the examiner provided an abstract version of the rule and if necessary an explicit version of the rule. Sixty seconds were allowed for each sorting attempt in the cued sorting phase of the text.

Multiple measures of performance were derived from the CCST. For the free sorting phase, the total number of sorts attempted provided a measure of initiation. This total score was broken down into three additional measures: (1) the number of correct sorts, (2) the number of perseverative sorts (i.e., sorting the cards according to the same rule a second or third time), and (3) the number of other errors (e.g., sorting the cards into piles with unequal numbers of cards). The quality of the verbal explanations for the sorts correctly achieved was scored on a three-point scale as follows: two points were assigned if the subject verbalized the sorting principle that applied to both piles (e.g., "Color of the cards" or "These are green and these are yellow"); one point was assigned if the subject verbalized the rule as it applied to only one of the piles (e.g., "These are green and these aren't"); zero points were assigned for "don't know" responses and for responses that were clearly incorrect. The number of verbal perseverations (i.e., restating a previously given rule) was also counted. The structured sorting phase was scored exactly as was the verbal portion of the free sorting phase. In the cued sorting phase, the numbers of correct sorts in response to abstract cues and to explicit cues were analyzed separately.

\section{RESULTS}

Table 1 summarizes the demographic characteristics of the three age groups as well as their performance on the WCST. As can be seen in the table, the average education levels were high but comparable for the three groups.

As expected, the old group performed more poorly on the WCST than did either the young or the middle-aged groups. Significant differences were observed on the number of categories achieved and the number of perseverative responses and errors, but the groups did not differ in the number of nonperseverative errors, trials to the first cate- 
gory, or failures to maintain set. Note also that the performance of subjects in the old group was considerably more variable than that of subjects in the other groups. The magnitude of within-group variance was 5.64 times as great for the old subjects as for the middle-aged on the number of categories achieved. For perseverative responses, the ratio of within-group variances for these two groups was 7.93.

Table 2 summarizes the results on the CCST. In the free sorting condition, the old group attempted fewer sorts and achieved fewer correct sorts than did the young or the middle-aged group, but the old subjects did not make more verbal or nonverbal perseverative errors, nor did they commit more nonperseverative (other) sorting errors. When sorting performance was expressed as a percentage of total sorts, no significant differences among groups were observed.

The subjects in the old group attained fewer total points for their verbal explanations than did the subjects in the other two groups. However, since verbal points are awarded only for explanations of correct sorting responses, points/correct sort provides a purer measure of the quality of verbal explanations. On this measure, the old subjects scored slightly, but not significantly higher than subjects in the young or the middle-aged group.

In the structured sorting condition, the old subjects attained fewer total points (indicating that they identified fewer concepts) and made more perseverative errors than did the subjects in the other two groups. The old subjects also performed more poorly than the other subjects in the abstract condition of the cued sorting phase. No significant differences were observed in the explicit condition, but most subjects in all groups attained maximum (24) scores.

Examination of the relative magnitudes of within-group variances indicated that, with one exception, the ratios of within-group variances for the old versus the middleaged group were less than 2.0. For perseverative responses during the structured sorting phase, the ratio of within-group variances for these two groups was 3.01.

There were no significant differences between means or variances for the young and middle-aged groups on any measure.

Examination of patterns of correlations for various measures revealed the usual high and negative relationship between the number of categories achieved on the WCST and the number of perseverative responses and errors (absolute $r \mathrm{~s}>.85, \mathrm{ps}<.001)$. For the free sorting phase of the CCST, correlations between measures of concept attainment and perseveration were low and statistically insignificant (absolute $r \mathrm{~s}<.22$ ). For the structured sorting phase, however, the number of points attained was negatively correlated with the number of perseverations $(r=-.65, p<.001)$.

Despite the wide range of education of the subjects (8-20 years), there were no significant relationships between education and any of the measures of performance obtained on the CCST (absolute $r s<.17$ ). These results should be interpreted cautiously, however, because level of education was not significantly correlated with any of the measures of performance from the WCST $(a b-$ solute $r$ s $<.20$ ).

\section{DISCUSSION}

The present findings reveal that the CCST, like most tests of problem solving (Heaton et al., 1986; Salthouse, 1992), is sensitive to the effects of age. Consideration of the pattern of the age differences on the CCST, however, helps to isolate the nature of the problem-solving deficiencies in this highly educated normal elderly sample.

Results of the free sorting phase indicated that the elderly group generated fewer correct sorts and fewer total sorts than did the young and middle-aged groups; but they showed no increased tendency to make verbal or nonverbal perseverative responses and they explained their correct sorting responses as well as the younger subjects did. Since the free sorting phase is timed, one might attribute the deficits of the older subjects primarily to a simple slowing of mental and/or motor responding (see, e.g., Charness, 1985).

Consideration of performance on the structured and cued phases of the CCST indicates that such an interpretation is probably incomplete. During these phases, the $60 \mathrm{sec}$ allowed for responding were almost never used, so it is unlikely that impairments can be attributed to mental slowing. Instead, the fact that the old group attained fewer points on the structured sorting phase and abstract portion of the cued sorting phase suggests that they were less able to identify concepts and to follow abstractly stated rules. The increased number of perseverative responses by the old group during the structured sorting phase probably reflects a genuine increased tendency to perseverate under some circumstances, although the increased perseverative responding observed on this measure may simply be a secondary consequence of poorer overall performance. The latter interpretation is consistent with the high negative correlation between points attained and perseverative responses that was observed only on the structured sorting phase.

One deterrent to the widespread use of the CCST in clinical practice is the time (45-60 $\mathrm{min}$ ) required for one to administer the complete (i.e.,

Table 1

Demographic Characteristics and Performance on the Wisconsin Card Sorting Test

\begin{tabular}{|c|c|c|c|c|c|c|c|}
\hline & \multicolumn{2}{|c|}{ Young } & \multicolumn{2}{|c|}{ Middle Aged } & \multicolumn{2}{|c|}{ Old } & \multirow{2}{*}{$\begin{array}{c}F(2,62) \\
\text { for Groups } \\
\end{array}$} \\
\hline & $M$ & $S D$ & $M$ & $S D$ & $M$ & $S D$ & \\
\hline Age & 25.5 & 5.7 & 40.6 & 6.1 & 70.9 & 6.5 & $306.71 \ddagger$ \\
\hline Education & 15.0 & 2.2 & 15.8 & 2.5 & 15.5 & 4.2 & 0.41 \\
\hline Categories & 5.8 & 0.7 & 5.8 & 0.8 & 4.6 & 1.98 & $6.63+$ \\
\hline Perseverative responses & 11.3 & 7.5 & 11.0 & 9.8 & 24.2 & 27.68 & $4.02 *$ \\
\hline Perseverative errors & 9.9 & 5.8 & 9.9 & 8.5 & 20.5 & 21.58 & $4.34 *$ \\
\hline Nonperseverative errors & 10.1 & 7.6 & 7.7 & 8.1 & 13.0 & 9.4 & 2.12 \\
\hline Trials to 1st category & 13.3 & 5.2 & 13.5 & 6.6 & 18.8 & 25.6 & 0.88 \\
\hline Failure to maintain set & 0.6 & 1.1 & 0.5 & 0.7 & 1.1 & 1.4 & 2.07 \\
\hline
\end{tabular}

${ }^{*} p<.05 . \quad \dagger p<.01 . \quad \ddagger p<.001 . \quad$ §Differs significantly $(\mathrm{p}<.05$ ) from young and middle-aged groups by Fisher LSD test. 
Table 2

Performance on the California Card Sorting Test

\begin{tabular}{|c|c|c|c|c|c|c|c|}
\hline & \multicolumn{2}{|c|}{ Young } & \multicolumn{2}{|c|}{ Middle Aged } & \multicolumn{2}{|c|}{ Old } & \multirow{2}{*}{$\begin{array}{c}F(2,62) \\
\text { for Groups }\end{array}$} \\
\hline & $M$ & $S D$ & $M$ & $S D$ & $M$ & $S D$ & \\
\hline \multicolumn{8}{|c|}{ Free Sorting } \\
\hline Total sorts & 20.3 & 2.4 & 20.6 & 2.9 & 18.2 & $3.0 \dagger$ & $4.69 *$ \\
\hline Correct sorts & 18.3 & 1.9 & 18.1 & 2.2 & 15.9 & $3.0 \dagger$ & $6.70 \dagger$ \\
\hline Perseverative sorts & 1.5 & 1.6 & 1.9 & 1.8 & 2.0 & 1.8 & 0.50 \\
\hline Other errors & 0.5 & 0.8 & 0.6 & 0.6 & 0.3 & 0.6 & 0.75 \\
\hline$\%$ Correct & 90.7 & 6.5 & 88.4 & 7.9 & 87.4 & 9.5 & 0.95 \\
\hline$\%$ Perseverative sorts & 7.1 & 6.7 & 8.9 & 7.5 & 10.8 & 9.0 & 1.18 \\
\hline$\%$ Other & 2.2 & 3.7 & 2.7 & 3.0 & 1.8 & 3.3 & 0.39 \\
\hline \multicolumn{8}{|c|}{ Verbal } \\
\hline Total points & 32.5 & 4.1 & 32.0 & 4.7 & 28.9 & $6.1 \neq$ & $3.31^{*}$ \\
\hline Points/correct & 1.77 & 0.1 & 1.77 & 0.1 & 1.82 & 0.1 & 1.09 \\
\hline Perseverative responses & 0.1 & 0.3 & 0.3 & 0.8 & 0.2 & 0.5 & 0.63 \\
\hline \multicolumn{8}{|c|}{ Structured Sorting } \\
\hline Total points & 32.2 & 6.8 & 30.1 & 7.0 & 26.1 & $8.2 \ddagger$ & $3.81 *$ \\
\hline Perseverative responses & 0.5 & 0.9 & 0.8 & 1.9 & 2.3 & $3.3 \ddagger$ & $4.05^{*}$ \\
\hline \multicolumn{8}{|c|}{ Cued Sorting } \\
\hline Abstract & 22.2 & 1.0 & 21.7 & 1.5 & 21.0 & $1.3 \ddagger$ & $4.68 *$ \\
\hline Explicit & 23.8 & 0.4 & 23.8 & 0.5 & 23.5 & 0.9 & 1.55 \\
\hline
\end{tabular}

three-set) version of the test that was used in this study. However, in our recent study of problem solving by schizophrenic patients (Beatty et al., in press), we found that a short version, consisting of the first set, was nearly as sensitive as the longer version. It will be important to determine whether or not the short version of the CCST is also sensitive to deficits in problem solving exhibited by less severely impaired patient groups.

\section{REFERENCES}

Beatty, W. W., Jocic, Z., Monson, N., \& Katzung, V. M. (in press). Problem solving by schizophrenic and schizoaffective patients on the Wisconsin and California Card Sorting Tests. Neuropsychology.

Beatty, W. W., Katzung, V. M., Nixon, S. J., \& Moreland, V. J. (in press). Problem solving deficits in alcoholics: Evidence from the California Card Sorting Test. Journal of Studies on Alcohol.

Beatty, W. W., \& Monson, N. (1990). Problem solving in Parkinson's disease: Comparison of performance on the Wisconsin and California Card Sorting Tests. Journal of Geriatric Psychiatry \& Neurology, 3, 163-171.

BeatTy, W. W., \& Monson, N. (in press). Problem solving by patients with multiple sclerosis: Comparison of performance on the Wisconsin and California Card Sorting Tests. International Journal of Clinical Neuropsychology.

Charness, N. (1985). Aging and problem-solving performance. In N. Charness (Ed.), Aging and human performance (pp. 225-259). New York: Wiley.

Davis, H. P., Cohen, A., Gandy, M., Colombo, P., Van DusselDORP, G., Simolke, N., \& Romano, J. (1990). Lexical priming deficits as a function of age. Behavioral Neuroscience, 104, 288-297.
Delis, D. C., Bihrle, A. M., Janowsky, J. S., Squire, L. R., \& Shimamura, A. P. (1989). Fractionation of problem-solving deficits in frontal-lobe patients. Journal of Clinical \& Experimental Neuropsychology, 11, 50.

Delis, D. C., SQuire, L. R., Bihrle, A., \& Massman, P. (1992). Componential analysis of problem-solving ability: Performance of patients with frontal-lobe damage and amnesic patients on a new sorting test. Neuropsychologia, 30, 683-698.

Folstein, M. F., Folstein, S. E., \& McHugh, P. R. (1975). "MiniMental State": A practical method of grading the cognitive state of patients for the clinician. Journal of Psychiatric Research, 12, 189-198.

Heaton, R. K. (1981). Wisconsin Card Sorting Test manual. Odessa, FL: Psychological Assessment Resources.

Heaton, R. K., Grant, I., \& Matthews, C. C. (1986). Differences in neuropsychological test performance associated with age, education, and sex. In I. Grant \& K. M. Adams (Eds.), Neuropsychological assessment of neuropsychiatric disorders (pp. 100-120). New York: Oxford University Press.

Heaton, R. K., Grant, I., \& Matthews, C. C. (1991). Comprehensive norms for an expanded Halstead-Reitan battery: Demographic corrections, research findings and clinical applications. Odessa, FL: Psychological Assessment Resources.

Reitan, R. M., \& Wolfson, D. (1985). The Halstead-Reitan Neuropsychological Test Battery. Tucson, AZ: Neuropsychology Press.

SAlthouse, T. A. (1992). Reasoning and spatial abilities. In F. I. M. Craik \& T. A. Salthouse (Eds.), The handbook of aging and cognition (pp. 167-212). Hillsdale, NJ: Erlbaum.

(Manuscript received May 27, 1993.) 